\title{
A Comparison of Survival after Radiosurgery in Non- Small Cell Lung Cancer Patients with One versus More than Twenty Brain Metastases
}

\section{Zhishuo Wei}

UPMC: University of Pittsburgh Medical Center https://orcid.org/0000-0002-9091-1734

Ajay Niranjan ( $\square$ niraax@upmc.edu )

UPMC: University of Pittsburgh Medical Center https://orcid.org/0000-0001-6754-8597

Hussam Abou-Al-Shaar

UPMC: University of Pittsburgh Medical Center

Hansen Deng

UPMC: University of Pittsburgh Medical Center

\section{Luigi Albano}

UPMC: University of Pittsburgh Medical Center

\section{Dade Lunsford}

UPMC: University of Pittsburgh Medical Center

\section{Research Article}

Keywords: stereotactic radiosurgery, non-small cell lung cancer, brain metastasis, adverse radiation effect

Posted Date: December 9th, 2021

DOl: https://doi.org/10.21203/rs.3.rs-887529/v1

License: (1) This work is licensed under a Creative Commons Attribution 4.0 International License.

Read Full License 


\section{Abstract \\ Background}

Whether the number or cumulative volume of brain metastases affects survival in patients with metastatic non-small cell lung cancer (NSCLC) remains controversial. We sought to compare whether patients with solitary brain disease had better outcomes than patients with $\geq 20$ brain metastases.

\section{Methods}

Between 2014 to 2020, 26 NSCLC patients (925 tumors) underwent stereotactic radiosurgery (SRS) for $\geq$ 20 metastases in a single procedure (median margin dose $=16 \mathrm{~Gy}$, median cumulative tumor volume $=$ $4.52 \mathrm{cc}$ ); 56 patients underwent SRS for a single metastasis (median margin dose $=18 \mathrm{~Gy}$, median volume $=4.74 \mathrm{cc}$ ). The overall survival $(\mathrm{OS})$, local tumor control $(\mathrm{LC})$, adverse radiation effect (ARE) risk, and incidence of new tumor development were compared.

\section{Results}

No difference in OS was found between patients with $\geq 20$ brain metastases (median OS $=15$ months) and patients with solitary metastasis (median OS $=12$ months; $p=0.3$ ). In the solitary tumor cohort, two of $56(3.5 \%)$ tumors progressed whereas in the $\geq 20$ cohort only 3 of $925(0.3 \%)$ tumors showed progression ( $\left.{ }^{*} p=0.0013\right)$. The rate of new tumor development was significantly higher in patients with $\geq$ 20 tumors $\left({ }^{\star \star *} p=0.0001\right)$. No significant difference of ARE rate was found $(7.5 \%$ for $\geq 20$ tumors vs. $8.7 \%$ for single metastasis).

\section{Conclusions}

Patients with $\geq 20$ tumors showed significantly better LC with similar OS compared to patients with solitary tumors. Current guidelines that restrict the role of SRS to patients with 1-4 tumors should be revised.

\section{Introduction}

Lung cancer is one of the most common malignancies in the world, accounting for $11.4 \%$ of all documented cancer cases and $18 \%$ of total deaths [1]. Non-small cell lung cancer (NSCLC) is the most common subtype and $30 \%-40 \%$ of NSCLC patients who are screened for brain spread at the time of the primary diagnosis are found to have Stage IV disease [2, 3].

Historically, management approaches include both whole brain fractionated radiation therapy (WBRT) and surgical resection in patients who present with symptomatic but limited disease. Over the last two 
decades accumulating evidence showed that stereotactic radiosurgery (SRS) provides a non-invasive management option for many patients with brain metastatic spread, thereby sparing both the short and long term risks and failure rates of WBRT [4]. The local tumor control after SRS in NSCLC patients consistently exceeds $80 \%$ in the literature $[5,6]$.

Debate continues as to whether the number or the cumulative volume of brain metastases (BM) is more important to determine patients' eligibility and tumor response to brain SRS. Current training biases, national guidelines, and third-party insurance reimbursement approvals often determine which option patients can receive [7]. The present study evaluates whether cumulative tumor volume or number ( $1 \mathrm{vs} \geq$ 20) affects survival (OS) after SRS. We also assessed local control (LC), adverse radiation effect (ARE) incidence, and new tumor development rates in this volume matched two cohort comparison.

\section{Methods}

\section{Inclusion criteria and SRS treatment}

The details of SRS procedure have been documented in the previous publications [8-10]. Between 2014 and 2020, 82 NSCLC with either solitary brain metastasis or more than 20 brain metastases underwent SRS. Twenty-six patients with $\geq 20 \mathrm{BMs}$ (cumulative tumor volume $=4.52 \mathrm{cc}$ ) were volume matched with 56 patients with solitary tumors (cumulative tumor volume $=4.74 \mathrm{cc}$ ). All patients had a Karnofsky performance Score (KPS) of $\geq 70$. The histological sub-types of the primary lesion, extent of extracranial metastatic disease, previous brain surgery or WBRT, systemic treatment, and the number of BM were retrospectively reviewed from patients' medical record or radiographic imaging. On the day of SRS, mild sedation with intravenous midazolam and fentanyl along with local anesthetic were used for frame placement. High-resolution contrasted stereotactic magnetic resonance imaging (MRI) was performed. T1-weighted sequence with 1.5-mm slice thickness that was used for target localization and treatment planning. SRS was performed using the Leksell Gamma Knife (AB Elekta, Stockholm, Sweden) [8]. Tumor margin doses ranging from 16 to 20 Gy were prescribed based on tumor volume, location, and histology. Lower doses were used for larger tumor volumes or in cases with adjacent critical structures such as the brainstem, optic nerves, or cochlea.

\section{Patient Characteristics}

For the 26 patients with $\geq 20 \mathrm{BMs}$, the median age was 64 years with females and males constituting $57 \%$ and $43 \%$, respectively. The most common histological type was adenocarcinoma (77\%). Nearly eight percent of the patients had received prior WBRT, and 15\% of patient had prior craniotomy to remove one or more BMs. Eighty five percent of patients received chemotherapy and $61.5 \%$ of patients received immunotherapy. The median number of metastases treated was 25 , and the average total tumor volume per patient was $4.52 \mathrm{cc}$. During SRS a median margin doe of $16 \mathrm{~Gy}$ (range from $13-20 \mathrm{~Gy}$ ) was prescribed to a median of $80 \%$ isodose. The a median maximum dose received by each tumor of $20 \mathrm{~Gy}$ and the median total $12 \mathrm{~Gy}$ volume was $22.52 \mathrm{cc}$. 
The median age for the 57 NSCLC patients with solitary tumors was 57 years, ranging from $44-84$ years old (Table 1), with 1:1 female to male ratio. Adenocarcinoma (65\%) and squamous cell carcinoma $(17.5 \%)$ represented the most common histological types. None of the patients received prior WBRT, and $28 \%$ of patient had prior surgical resection of tumor followed by tumor bed SRS. Most patients $(83 \%)$ received chemotherapy and $17.5 \%$ received immunotherapy. The median tumor volume was $4.74 \mathrm{cc}$ (ranging from $0.62 \mathrm{cc}$ to $29.65 \mathrm{cc}$ ). During SRS the median $12 \mathrm{~Gy}$ volume was $13.41 \mathrm{cc}$ and the median maximum dose received by each tumor was $35.5 \mathrm{~Gy}$. 
Table 1

Summary of clinical characteristics of 83 NSCLC BM patients

\begin{tabular}{|c|c|c|c|c|}
\hline \multirow{2}{*}{$\begin{array}{l}\text { Characteristics } \\
\text { Patients, n }\end{array}$} & \multirow{2}{*}{$\begin{array}{l}\text { Total } \\
82\end{array}$} & \multicolumn{2}{|c|}{$\begin{array}{l}\text { Number of tumors } \\
\geq 20 \text { tumors } 1 \text { tumor }\end{array}$} & \multirow[t]{2}{*}{$p$ values } \\
\hline & & 26 & 56 & \\
\hline \multicolumn{5}{|l|}{ Tumor numbers } \\
\hline Median & & 25 & 1 & \\
\hline Range & $1-64$ & $21-64$ & 1 & \\
\hline \multicolumn{5}{|l|}{ Age, years } \\
\hline Median & & 64 & 67 & 0.37 \\
\hline Range & $43-84$ & $43-83$ & $44-84$ & \\
\hline \multicolumn{5}{|l|}{ Sex 0.35} \\
\hline Male & 39 & $11(43)$ & $28(50)$ & \\
\hline Female & 43 & $15(57)$ & $28(50)$ & \\
\hline \multicolumn{5}{|l|}{ Histology 0.13} \\
\hline Adenocarcinoma & 58 & $20(77)$ & $38(68)$ & \\
\hline Squamous cell & 11 & $0(0)$ & $11(20)$ & \\
\hline Large cell & 6 & $3(11.5)$ & $3(5)$ & \\
\hline Undifferentiated & 7 & $3(11.5)$ & $4(7)$ & \\
\hline \multicolumn{5}{|c|}{ Chemotherapy 0.72} \\
\hline Yes & 69 & $22(85)$ & $47(84)$ & \\
\hline \multicolumn{5}{|c|}{ Immunotherapy 0.16} \\
\hline Yes & 26 & $16(61.5)$ & $10(18)$ & \\
\hline Prior WBRT & & & & 0.16 \\
\hline Yes & 2 & $2(7.5)$ & $0(0)$ & \\
\hline \multicolumn{5}{|l|}{ Prior surgery 0.15} \\
\hline Yes & 20 & $4(15)$ & $16(28.5)$ & \\
\hline \multicolumn{5}{|l|}{ Tumor volume (cc) } \\
\hline Median & & 4.52 & 4.74 & 0.82 \\
\hline Range & $0.58-32.49$ & $0.58-32.49$ & $0.62-29.65$ & \\
\hline
\end{tabular}




\begin{tabular}{|c|c|c|c|c|}
\hline Characteristics & Total & \multicolumn{2}{|c|}{$\begin{array}{l}\text { Number of tumors } \\
\geq 20 \text { tumors } 1 \text { tumor }\end{array}$} & p values \\
\hline \multicolumn{5}{|c|}{12 Gy volume (cc) } \\
\hline Median & & 22.52 & 13.41 & $0.002^{\star}$ \\
\hline Range & $2.26-92.5$ & $2.49-92.5$ & $2.26-5$ & \\
\hline \multicolumn{5}{|l|}{ Margin dose (Gy) } \\
\hline Median & & 18 & 18 & 0.13 \\
\hline Range & $19.9-40$ & $15-20$ & $13-20$ & \\
\hline \multicolumn{5}{|c|}{ Values in parentheses are percentages } \\
\hline \multicolumn{5}{|c|}{$\begin{array}{l}\text { Based on } 147 \text { patients ( } 115 \text { in group } A \text { and } 32 \text { in group } B ; 65 \text { patients were excluded because } \\
\text { neuroimaging results were not available). }\end{array}$} \\
\hline
\end{tabular}

\section{Study End Points}

OS, local control (LC), the rate of new tumor development, and rate of ARE after SRS were determined. OS was defined from the time of the first SRS procedure until the last documented visit in the medical record, or the time of death. Progression was documented when treated tumor showed sustained enlargement on follow-up imaging. New tumor development was documented when a new MRI enhancing lesion was noted on follow-up imaging compared to SRS planning scan. ARE was diagnosed based on either MRI analysis including T1/T2 mismatch as described by Kano et al. [11] or surgical pathological confirmation of radiation necrosis. Suspicious but indeterminate contrast enhancements (tumor recurrence vs ARE) were followed up with MRI until local recurrence or ARE could be discerned.

\section{Statistical Analyses}

Statistical analyses were performed with Prism version 9 (GraphPad, California). Survival, local control, regional control, and adverse radiation effect curves were generated using the Kaplan-Meier method. A $p$ value of $<0.05$ was necessary to be considered statistically significant. Patients with solitary BM whose volume was less than $0.5 \mathrm{cc}$ were excluded from this study to control the total tumor volume between the two patient groups.

\section{Results}

\section{Survival Rates}

The median OS for patients with solitary BM was 12 months. By comparison, the median survival for patients with $\geq 20$ BMs was 15 months. No statistically significant difference in survival rates was observed ( $p=0.3$ ) between the two cohorts (Fig. 1). 


\section{Local Failure, Regional Failure, and Adverse Radiation Effect}

For patients with $\geq 20 \mathrm{BMs}, 3$ tumors out of 925 treated tumors $(0.3 \%)$ had documented local tumor progression at a median time to local progression of 8 months after the first SRS procedure (range $2-10$ months). In comparison, for patients with solitary BM, 2 tumors out of 56 tumors (3.5\%) had a documented tumor progression at a median time of 9 months (range $5-13$ months). In this tumor volumetric matched comparison, patients with multiple small BMs showed statistically significant improved tumor control rate compared to patients with solitary metastasis $(p=0.0013)$ (Fig. 1B-C).

New tumor development occurred in 17 (65\%) patients with $\geq 20$ BMs. The median time until new tumor development was 6 months (range 2-45 months). In contrast eight (14\%) patients with solitary tumors patients 8 out of 56 patients (14\%) had new disease at an 8 month median (range 2-53 months). Patients with $\geq 20$ BMs have a significantly higher ( $* \star x p=0.0001$ ) rate of new tumor development compared to patients with solitary BM, possibly related to a higher rate of active systemic disease in patients with multiple BMs (Fig. 1D).

The risk of ARE was $3.8 \%$ for patients with $\geq 20$ metastases comparted to $8.7 \%$ for patients with solitary BM. A single patient with $\geq 20$ BMs developed ARE 9 months after initial SRS. This asymptomatic patient was managed with vitamin E and Trental for 90 days until the MRI reactive changes resolved. In contrast, 5 patients $(8.7 \%)$ with solitary BM developed ARE. Three received immunotherapy prior to SRS, and all were clinically symptomatic. The median onset of ARE was 6 month after the SRS, ranging from 2 to 29 months.

\section{Discussion}

SRS is a safe and effective management modality for the CNS metastases from NSCLC. Bowden et al. reported that the median survival of NSCLC patients with brain metastasis was 19.9 months, with 1-year survivals of $70 \%$ and 5 -year survival rates of $16 \%$ [9]. The initial management of patients with both solitary and multiple management of metastatic NSCLC continues to evolve, despite past guidelines that only patients with 1-4 brain metastases are considered appropriate for initial treatment by SRS. In the current study, we sought to determine whether tumor number or total tumor volume can serve as a better predictive factor for patients' survival after SRS.

Although high definition MRI can determine the number of brain metastases, simple tumor number is insufficient to determine the best strategy. At present neither medical oncologists, radiation oncologists, or neurosurgeons are able to provide accurate predictions of survival when confronted with a new patient with metastatic brain disease [12]. Routman et al. [13] found that the number of brain metastases was not a significant predictor of patient survival for patients who underwent SRS with or without prior WBRT and/or surgery. Chang et al. [14] reviewed 323 brain metastasis patients who underwent SRS and found no significant difference in survivals among patient groups that had $1-5,6-10,11-15$, and $\geq 15 \mathrm{BMs}$. 
Prior to the current analysis, we hypothesized that tumor volume was more prognostically important than the number of brain metastases. We found that the median survival in patients with $\geq 20$ metastases were statistically no different than the survival of patients with single metastases $(p=0.3)$. Our experience compares favorably with other outcome reports in current management of NSCLC patients. In 2013, Rava et al. [15] reported a median survival of 6.5 months in 53 patients with $\geq 10$ metastatic brain lesions. Chang and colleagues [14] reported that the median survival times for patients with 11-15 and $\geq$ 15 BMs was 13 months and 8 months, respectively. Karlsson et al. [16] analyzed 1921 metastatic patients who underwent SRS over the time span of 30 years and found no statistically significant survival differences among individuals with $2,3-4,5-8$, or $\geq 8$ metastases.

The model constructed by Ali et al. [17] found brain metastasis number as a continuous variable and only a $4 \%$ increase in the hazard of death for every increment of 6-7 metastases. This study showed that the number of brain metastases only serves as a modest prognostic factor in predicting patient survival and treatment recommendations should be evaluated using other factors when trying to decide between in the greater clinical context in the decision making between SRS and WBRT. While tumor number is often evaluated, cumulative tumor volume is rarely included in guidance documents [18].

In the context of active systemic cancer, some patients will experience new tumor development, progression of treated disease, or treatment related side effects. The present study showed a median onset of 8 months until the development of new tumors in patients with initially solitary tumors. In comparison, the interval between SRS and new tumor detection in the $\geq 20$ cohort was slightly less at 8 months. Somewhat surprisingly we found that median survivals were slightly longer in the $\geq 20$ cohort, although this finding was not statistically significant. In such patients additional salvage SRS can be considered along with the clinical symptoms, signs, Karnofsky performance status (KPS), prior WBRT treatment, extracranial disease status, absence of neurological symptoms, and patient's preference, among others.

Even patients who present with $\geq 20$ brain metastases can have survivals comparable to patients who have a single tumor of comparable cumulative volume. Active systemic disease status, neurological function in part measured by KPS scores remain important prognostic factors for both survival and new tumor development.

\section{Limitations}

The current study has limitations previously described for small, nonrandomized retrospective reports. Thus, the results presented here may not be extrapolated to all patients and all types of metastatic cancer. Patients who develop new untreated disease must be reassessed for additional salvage SRS or other options. Metastatic brain disease requires individual patient careful consideration to develop an appropriate care plan.

\section{Conclusions}


SRS is an effective management option for patients with 20 or more BMs. Overall survival of patients with 20 or more metastases was no different than survivals in NSCLC patients who had with single BM if the cumulative tumor volume was controlled total tumor volume. This suggests that total tumor volume may be more predictive of survival than number of brain metastases.

\section{Declarations}

Funding: The authors declare that no funds, grants, or other support were received during the preparation of this manuscript.

Competing Interests: Dr. L. Dade Lunsford is an AB Elekta stockholder. All other authors have no competing interests to declare that are relevant to the content of this article.

Author contributions: Ajay Niranjan and L. Dade Lunsford designed the study. Material preparation, data collection and analysis were performed by Zhishuo Wei, Hussam Abou-Al-Shaar, Hansen Deng and Luigi Albano. The first draft of the manuscript was written by Zhishuo Wei and all authors commented on previous versions of the manuscript. All authors read and approved the final manuscript.

Data availability: The datasets generated during and/or analyzed during the current study are available from the corresponding author on reasonable request.

Ethical approval: All procedures performed in studies involving human participants were in accordance with the ethical standards of the institutional and/or national research committee and with the 1964 Helsinki declaration and its later amendments or comparable ethical standards. The study (IRB: STUDY20010256) was approved by the Ethical Committee at the University of Pittsburgh Medical Center.

Consent to participate: This study (IRB: STUDY20010256) was exempt from consent from participants as it involved the analysis of medical records only.

Consent to publish: The authors affirm that human research participants' consent for publication of the images in Figure(s) 1 (a-d), and 2 (a-d) was not needed as the data is anonymized.

\section{References}

1. Sung, H., et al., Global Cancer Statistics 2020: GLOBOCAN Estimates of Incidence and Mortality Worldwide for 36 Cancers in 185 Countries. CA Cancer J Clin, 2021. 71(3): p. 209-249.

2. Siegel, R.L., et al., Cancer Statistics, 2021. CA Cancer J Clin, 2021. 71(1): p. 7-33.

3. Linskey, M.E., et al., The role of stereotactic radiosurgery in the management of patients with newly diagnosed brain metastases: a systematic review and evidence-based clinical practice guideline. $\mathrm{J}$ Neurooncol, 2010. 96(1): p. 45-68.

4. Halasz, L.M., et al., Use of stereotactic radiosurgery for brain metastases from non-small cell lung cancer in the United States. Int J Radiat Oncol Biol Phys, 2013. 85(2): p. e109-16. 
5. Mariya, Y., et al., Outcome of stereotactic radiosurgery for patients with non-small cell lung cancer metastatic to the brain. J Radiat Res, 2010. 51(3): p. 333-42.

6. Yamamoto, M., et al., Stereotactic radiosurgery for patients with multiple brain metastases (JLGK0901): a multi-institutional prospective observational study. Lancet Oncol, 2014. 15(4): p. 38795.

7. Brown, P.D., et al., Effect of Radiosurgery Alone vs Radiosurgery With Whole Brain Radiation Therapy on Cognitive Function in Patients With 1 to 3 Brain Metastases: A Randomized Clinical Trial. JAMA, 2016. 316(4): p. 401-409.

8. Bowden, G.N., et al., Clinical dose profile of Gamma Knife stereotactic radiosurgery for extensive brain metastases. J Neurosurg, 2020. 134(5): p. 1430-1434.

9. Bowden, G., et al., Gamma knife radiosurgery for the management of cerebral metastases from nonsmall cell lung cancer. J Neurosurg, 2015. 122(4): p. 766-72.

10. Bowden, G., et al., Gamma Knife Radiosurgery for the Management of More Than 15 Cerebral Metastases. World Neurosurg, 2019. 126: p. e989-e997.

11. Kano, H., et al., T1/T2 matching to differentiate tumor growth from radiation effects after stereotactic radiosurgery. Neurosurgery, 2010. 66(3): p. 486-91; discussion 491-2.

12. Kondziolka, D., et al., The accuracy of predicting survival in individual patients with cancer. J Neurosurg, 2014. 120(1): p. 24-30.

13. Routman, D.M., et al., The growing importance of lesion volume as a prognostic factor in patients with multiple brain metastases treated with stereotactic radiosurgery. Cancer Med, 2018. 7(3): p. 757764.

14. Chang, W.S., et al., Analysis of radiosurgical results in patients with brain metastases according to the number of brain lesions: is stereotactic radiosurgery effective for multiple brain metastases? $\mathrm{J}$ Neurosurg, 2010. 113 Suppl: p. 73-8.

15. Rava, P., et al., Survival among patients with 10 or more brain metastases treated with stereotactic radiosurgery. J Neurosurg, 2013. 119(2): p. 457-62.

16. Karlsson, B., et al., Thirty years' experience with Gamma Knife surgery for metastases to the brain. J Neurosurg, 2009. 111(3): p. 449-57.

17. Ali, M.A., et al., Survival Patterns of 5750 Stereotactic Radiosurgery-Treated Patients with Brain Metastasis as a Function of the Number of Lesions. World Neurosurg, 2017. 107: p. 944-951 e1.

18. Gorovets, D., et al., Multi-institutional Nomogram Predicting Survival Free From Salvage Whole Brain Radiation After Radiosurgery in Patients With Brain Metastases. Int J Radiat Oncol Biol Phys, 2017. 97(2): p. 246-253.

\section{Figures}




\section{Figure 1}

Survival curves and tumor control in patients with $\geq 20$ brain metastases and patients with solitary brain metastasis. (A) Overall survival in the subset of 83 case-matched and volume-matched patients according to tumor numbers. Twenty-six patients (red) with $\geq 20$ brain metastases and fifty-seven patients (blue) with solitary brain metastases were identified. (B) Local tumor control rate per tumor in patients with $\geq 20$ brain metastases vs. patients with solitary brain metastasis. (C) Local tumor control rate comparison calculated per patient. (D) Distant tumor control rate in patients with $\geq 20$ brain metastasis vs. patients with solitary brain metastases (* $p<0.05$; $* *<<0.01)$.
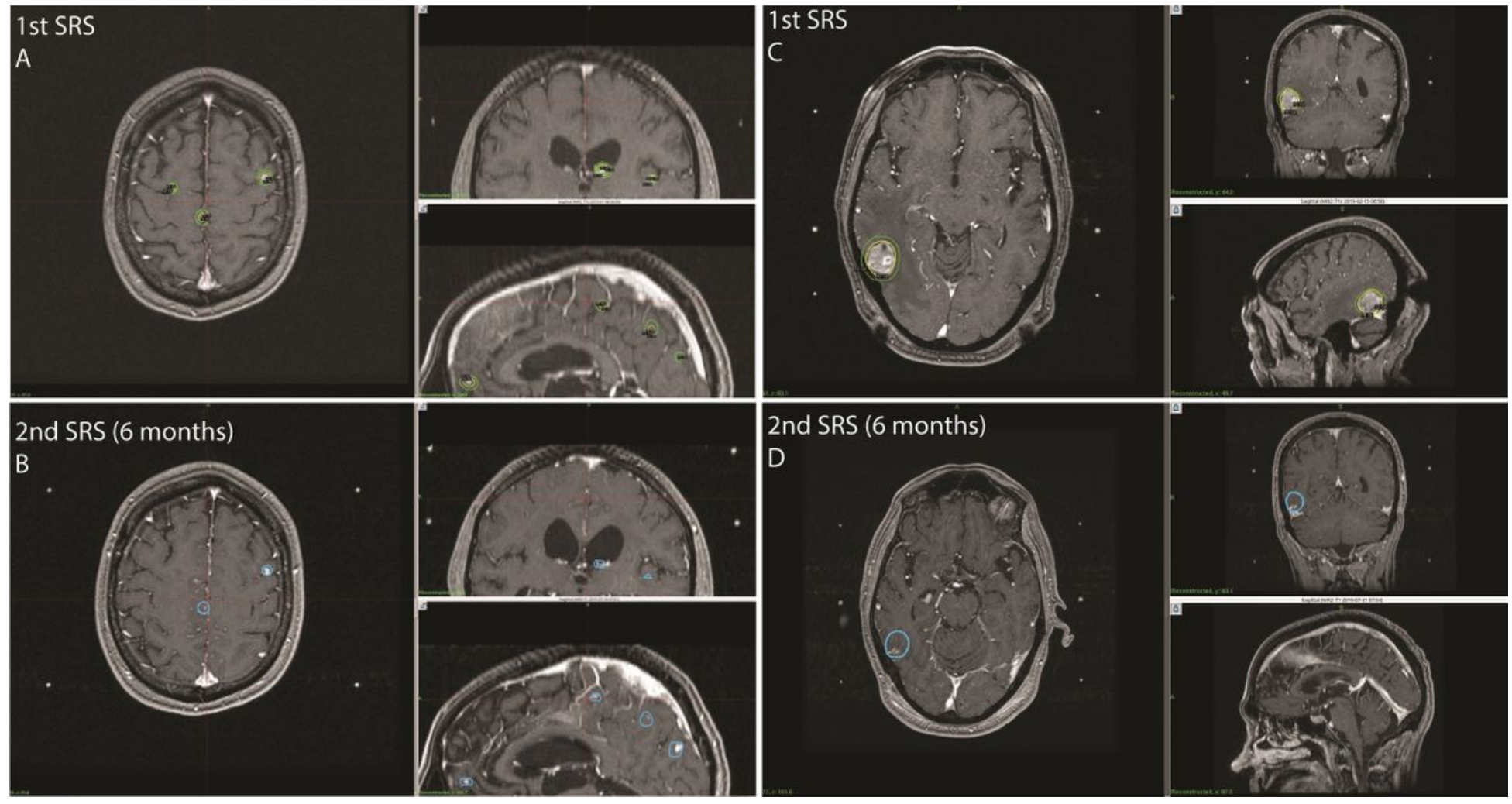

Figure 2

(A-B). A 65-year-old male patient with Stage IV non-small cell lung cancer (NSCLC) presented with 26 brain metastases and underwent SRS. Immediate tumor regression was seen 6 months following the first SRS treatment. Asymptotic distant tumor recurrence was seen on MRI 6 months after SRS and the patient had repeat SRS for his recurrent tumor, with subsequent complete tumor response. (C-D). A 76-year-old male patient with Stage IV NSCLC presented with solitary brain metastasis and underwent SRS as primary treatment. Six-months after the first SRS treatment, tumor regressed remarkably. 